Meta

Journal des traducteurs

Translators' Journal

\title{
Peut-on faire de la traduction juridique ? Comment doit-on l'enseigner?
}

\section{Michel Sparer}

Volume 47, numéro 2, juin 2002

Traduction et terminologie juridiques

URI : https://id.erudit.org/iderudit/008014ar

DOI : https://doi.org/10.7202/008014ar

Aller au sommaire du numéro

\section{Éditeur(s)}

Les Presses de l'Université de Montréal

\section{ISSN}

0026-0452 (imprimé)

1492-1421 (numérique)

Découvrir la revue

Citer cet article

Sparer, M. (2002). Peut-on faire de la traduction juridique ? Comment doit-on l'enseigner ? Meta, 47(2), 266-278. https://doi.org/10.7202/008014ar

\section{Résumé de l'article}

Cet article de 2002 est une occasion de formuler quelques considérations sur la compréhension de ce qu'est ou devrait être la traduction juridique et sur la formation des traducteurs et traductrices. Il s'agit de répondre à bien des questions ou préjugés : peut-on traduire des textes juridiques ? Faut-il être juriste pour le faire? Les cours de traduction juridique dispensés au premier cycle sont-ils suffisants pour qu'une personne puisse s'engager dans cette activité en tant que professionnel ? Le droit canadien est ici exprimé en deux langues. Mais le Canada et le Québec sont au confluent de deux traditions juridiques ; un contexte où l'on a deux vocabulaires juridiques : un vocabulaire juridique civiliste bilingue et un vocabulaire juridique bilingue de common law. Ce qui fait quatre vocabulaires juridiques. De plus, les langues évoluent : il existe autant de chances d'établir un lien parfait et stable entre deux termes que de passer d'une montgolfière à une autre en marchant sur une corde tendue entre les deux nacelles. Au delà de cette complexité, il est aussi essentiel d'amener les futurs traducteurs ou traductrices à comprendre ce qui se passe dans l'esprit du juriste qui a conçu le texte à traduire, selon la tradition juridique dont il est issu. Faut-il faire une maîtrise orientée en traduction juridique pour pouvoir toucher à ce domaine ? La formation en traduction juridique telle qu'on la connaît actuellement est-elle adéquate ? Malgré les préalables imposés pour l'accès à ce genre de cours, le programme de premier cycle en traduction devrait consacrer plus de temps à la traduction juridique.
Ce document est protégé par la loi sur le droit d'auteur. L’utilisation des services d'Érudit (y compris la reproduction) est assujettie à sa politique d'utilisation que vous pouvez consulter en ligne.

https://apropos.erudit.org/fr/usagers/politique-dutilisation/ 


\title{
Peut-on faire de la traduction juridique? Comment doit-on l'enseigner?
}

\author{
MICHEL SPARER \\ Directeur des Communications à l'Office des Professions du Québec, \\ Québec, Canada
}

\section{RÉSUMÉ}

Cet article de 2002 est une occasion de formuler quelques considérations sur la compréhension de ce qu'est ou devrait être la traduction juridique et sur la formation des traducteurs et traductrices. II s'agit de répondre à bien des questions ou préjugés : peut-on traduire des textes juridiques? Faut-il être juriste pour le faire? Les cours de traduction juridique dispensés au premier cycle sont-ils suffisants pour qu'une personne puisse s'engager dans cette activité en tant que professionnel?

Le droit canadien est ici exprimé en deux langues. Mais le Canada et le Québec sont au confluent de deux traditions juridiques; un contexte où l'on a deux vocabulaires juridiques: un vocabulaire juridique civiliste bilingue et un vocabulaire juridique bilingue de common law. Ce qui fait quatre vocabulaires juridiques. De plus, les langues évoluent: il existe autant de chances d'établir un lien parfait et stable entre deux termes que de passer d'une montgolfière à une autre en marchant sur une corde tendue entre les deux nacelles.

Au delà de cette complexité, il est aussi essentiel d'amener les futurs traducteurs ou traductrices à comprendre ce qui se passe dans l'esprit du juriste qui a conçu le texte à traduire, selon la tradition juridique dont il est issu. Faut-il faire une maîtrise orientée en traduction juridique pour pouvoir toucher à ce domaine? La formation en traduction juridique telle qu'on la connaît actuellement est-elle adéquate? Malgré les préalables imposés pour l'accès à ce genre de cours, le programme de premier cycle en traduction devrait consacrer plus de temps à la traduction juridique.

\section{ABSTRACT}

This article in 2002 provides the opportunity to formulate a few considerations regarding the comprehension of what is, or should be, legal translation and how translators are trained to cope with such translation. It is an attempt to address certain issues or biases found in questions such as: Can legal texts be translated? Should one be a trained legal specialist to do such translation? Are undergraduate university courses sufficient for preparing translators in this field?

Canadian law is expressed here in two languages. But Canada and Québec are home to two traditions of law, hence a context with two legal vocabularies: a bilingual civil law vocabulary and a bilingual common law vocabulary, in all four specialized vocabularies. Like all things, languages evolve. Finding a perfect and stable equivalence linking two terms in French and English is a daunting task that in the absolute, smacks of the impossible.

Besides systemic complexity, legal translators must understand the workings of the legal mind that composes texts to be translated and how this occurs in a specific legal tradition. Would a master's degree with a speciality in legal translation suffice? Despite the prerequisites for taking such a course, undergraduate translation courses should allot more time to legal translation.

\section{MOTS-CLÉS/KEYWORDS}

common law, Canada, formation des traducteurs juridiques, vocabulaire juridique 


\section{Introduction}

À l'origine du présent volume, on trouvera sans doute la conjonction deux traits de caractère : la vision du précurseur, celle de Wallace Schwab et le sens de la continuité, qui m'a poussé à lui proposer en 2000 de donner une suite à un numéro collectif de Meta sur le même sujet paru... en 1979 à l'initiative alors du professeur Jean-Claude Gémar. Notre démarche 23 ans plus tard porte à deux hypothèses: soit le sujet est riche... soit nous serions restés bloqués quelque part dans le passé, comme dans un film de Zemeckis. Nous préférons bien sûr la première hypothèse; la seconde nous rejoindra bien un jour.

En 1979, puis au fil de plus de 25 ans d'enseignement de la traduction juridique à l'Université, nous avons avancé l'idée que traduire des textes juridiques serait, en théorie du moins, un pari impossible. C'était le temps d'un débat qui portait à s'interroger, en 1981, sur la stéréophonie législative et les risques de la haute infidélité. Mais en même temps, nous nous sommes efforcés de tracer des routes pour surmonter ou pour contourner l'obstacle théorique.

"Il suivait son idée. C'était une idée fixe et il était surpris de ne pas avancer.» disait Jacques Prévert.

Chemin faisant, nous avons créé des manières de voir autrement, des outils pour comprendre, pour concevoir, pour exprimer et pour traduire des textes juridiques.

Restent bien sûr de grandes questions quant aux concepts, aux méthodes, que nous n'épuiserons pas non plus dans ce deuxième numéro thématique.

Cet article de 2002 est une occasion de mettre pied à terre et de formuler quelques considérations que nous croyons utiles sur la compréhension de ce qu'est ou devrait être la traduction juridique d'une part, et sur la question de la formation des traducteurs et traductrices d'autre part. Il s'agit de répondre à bien des questions ou préjugés: peut-on traduire des textes juridiques? Faut-il être juriste pour le faire? Les cours de traduction juridique dispensés au premier cycle sont-ils suffisants pour qu'une personne puisse s'engager dans cette activité en tant que professionnel?

Les points que nous évoquons sont là pour illustrer ce qui, à notre avis, devrait figurer dans un tel cours pour une formation ordonnée de nos étudiants et étudiantes. Par ailleurs, si notre propos est centré sur la traduction, nous sommes convaincus qu'il peut s'adresser aussi aux juristes qui sont associés à la préparation de la législation bilingue.

\section{Caractéristiques de la traduction juridique}

Attardons-nous à rappeler quel est le champ de cette activité et notamment ce qui caractérise la dualité juridique canadienne. Nous le ferons rapidement, certains autres articles évoquant déjà ces concepts. Notre article d'aujourd'hui n'est pas pour autant un cours de traduction juridique.

\section{A) Le champ de la traduction juridique}

En traduction juridique, on peut dénombrer cinq ou six domaines principaux.

D'abord, la législation. C'est-à-dire les lois et les règlements. Au Canada, cette activité touche la législation fédérale, mais aussi celle du Québec, du NouveauBrunswick ou du Manitoba et dans une certaine mesure, celles d'autres provinces ou 
territoires. En effet, la constitution du Canada prévoit que certains parlements doivent édicter leurs lois en français et en anglais. C'est une condition formelle de validité de la législation de ces parlements.

Ensuite, la traduction juridique s'applique à certains jugements. Notons au passage que lorsqu'on dit au Québec que la justice est bilingue, cela ne signifie pas que tout est traduit. Cela signifie simplement que chacun peut s'adresser aux tribunaux dans la langue officielle de son choix et que les juges peuvent, selon le cas, rendre leurs jugements en français ou en anglais. Toutefois, certains tribunaux comme la Cour suprême, traduisent systématiquement leurs jugements.

La traduction juridique s'attache également aux domaines des contrats, de forme plus libre, et qui en principe peuvent être rédigés en toute langue.

En nous éloignant un peu des textes qu'on pourrait qualifier de normatifs, il arrive qu'on ait à traduire des textes de doctrine, notamment des volumes ou des articles ou encore à traduire des documents à teneur juridique, des actes, des rapports ou des instruments divers.

Enfin, plus loin encore de la pratique classique de traduction juridique, on ne saurait oublier l'activité des interprètes qui agissent par exemple devant les tribunaux.

On le voit, la traduction juridique s'attache à plusieurs types de textes. Celui ou celle qui s'engage dans cette activité doit être conscient du fait que tous ces textes ne sont pas de même nature et qu'il faut en connaître les différences pour pouvoir accorder à chacun un traitement approprié.

La loi est en fait un texte assez pauvre, principalement articulé autour du «peut» et du «doit», passablement rigide et qui obéit à des habitudes et à un certain formalisme.

Le jugement est un texte plus discursif, au moins dans les pays de tradition britannique. On y décrit une situation pour faire ensuite une sorte de dissertation sur le droit qui aboutit à une décision.

Les textes contractuels pour leur part, ressemblent un tant soit peu à la loi en ce qu'ils énoncent essentiellement une série de règles, avec plus de latitude toutefois quant à la forme.

\section{B) Caractère de la dualité juridique canadienne}

Revenons un peu sur cette réalité, car il semble qu'elle ne soit pas toujours bien comprise. Dans l'esprit de plusieurs, la dualité canadienne, dans son expression juridique, se résume vaguement au bilinguisme des lois. La réalité est plus riche et plus complexe. Certes, le droit canadien est ici exprimé en deux langues. Mais il ne faut pas oublier pour autant que le Canada est lui-même au confluent de deux traditions juridiques et non pas seulement le rendez-vous, le point de contact de deux langues. Il est aussi et surtout le foyer de deux cultures principales. Ainsi quand on traduit de l'anglais au français, on change de langue mais on ne change pas de système juridique. On n'aboutira pas à faire d'un texte de common law rédigé en anglais un texte de droit civil rédigé en français. Il n'y a qu'une loi, même si elle s'exprime en deux langues. C'est de tout cela qu'il faut parler dans un cours de traduction juridique si on veut que nos étudiants et étudiantes sachent où ils mettent les pieds. 


\section{1) Deux systèmes juridiques}

Notre droit canadien et principalement notre droit québécois, sont en effet marqués par la coexistence de deux systèmes juridiques issus de deux traditions contrastées : le droit anglais ou common law et le droit français, aussi désigné à cette fin par les expressions droit romano-germanique ou tradition civiliste, selon les contextes ou les auteurs. Ces deux systèmes ou traditions illustrent avant tout deux façons de voir la société et la loi.

Dans le système britannique et pour schématiser, on voit le reflet d'une préférence pour une société libérale de droit immanent où la loi écrite tiendrait un rôle limité. Alors que dans le système juridique de type français, on conçoit que la loi puisse se mêler de régler assez largement la plupart des aspects de la vie.

Le système britannique fait naturellement une place importante aux tribunaux, gardiens des droits et libertés. La loi, dès sa conception, tient compte de l'interprétation que ces tribunaux pourraient lui donner; interprétation restrictive donc lorsque la loi restreint les droits ou établit des obligations, interprétation plus large lorsque la loi est attributive de droits et de garanties pour les libertés. Le rôle des tribunaux est donc important dans ce système.

Ce n'est pas la même situation en système français ou romano-germanique, où les tribunaux jouent un rôle moins actif et ne sont pas un «interlocuteur» aussi important pour le législateur. La jurisprudence n'y tient pas le même rang dans les sources du droit. Et si on peut, tout comme en système britannique, concevoir des réponses législatives à la jurisprudence, cela ne place pas pour autant les tribunaux de droit français sur le même pied d'influence que les tribunaux des régions de droit britannique. En terrain de droit français, le dialogue du législateur et du juge n'est pas du même niveau qu'en tradition juridique britannique. On constatera d'ailleurs qu'en France et dans l'Europe continentale en général, les jugements sont des textes assez lapidaires. Ils sont loin des développements beaucoup plus amples qu'on connaît ici en Amérique du Nord où l'on voit le juge exprimer plus généreusement ses idées, ses opinions, ses hésitations même, avant de conclure et trancher.

Voilà déjà une caractéristique importante qu'il convient de livrer à nos étudiants et étudiantes en traduction juridique. Tout cela mériterait bien sûr beaucoup de nuances, nuances que nous développons dans notre cours.

La loi est alors, pour schématiser, un peu comme un coffre à outils; les uns, de tradition française, considérant qu'il s'agit d'instruments qu'ils utilisent volontiers, de façon naturelle, alors que les autres, de tradition britannique, estiment qu'il s'agit d'outils dont on ne se sert qu'exceptionnellement, lorsqu'on y est obligé ; par exemple en cas de panne de courant. Il est certain que lorsque vient le moment de décrire ces outils, leur utilité, et de leur trouver une place dans l'atelier, cela fait une bonne différence. Toute comparaison est bien sûr boiteuse. C'est un peu la même chose lorsqu'il s'agit de savoir si on est en face d'un texte conçu comme un mode normal d'organisation sociale, appliqué — et donc interprété — de façon large (conception française) ou si, (comme en tradition britannique) on est en face d'un texte dont on veut limiter l'application en considérant la règle comme un remède à un problème particulier, un remède qui serait ponctuel, presque exceptionnel ou même anormal.

Il est clair que ces différences teinteront fortement la forme et la compréhension du texte de départ, notamment quant à sa portée et influenceront la façon de rédiger dans la langue d'arrivée. 
La façon empirique qu'a le système anglais d'accoler des règles ponctuelles et correctives, en se refusant à de grandes fresques «programmatoires» comme en français, produit des textes qui sont aussi différents que les jardins à l'anglaise le sont des jardins à la française. Le texte y est aussi libre — et parfois aussi tortueux — que la nature elle-même. Priorité à la réalité.

Une autre image peut illustrer cette différence. On a construit depuis 40 ans des campus universitaires étendus, avec de grandes avenues perpendiculaires prévues sans doute pour les voitures et qu'on a bordées de trottoirs pour les étudiants qui, eux, sont souvent à pied. Ils devraient donc suivre les rues, là où des sentiers plus directs, tout en diagonales, leur auraient permis de se rendre plus rapidement d'un pavillon à l'autre. On devine ce qui s'est produit: des sentiers se sont rapidement tracés dans l'herbe des terre-pleins rectangulaires; les piétons avaient naturellement décidé d'aller au plus court.

Sans vouloir caricaturer, observons ce qui se passe dans l'une et l'autre culture. Dans l'une, on tire les conséquences de la réalité et on réaménage les sentiers pour piétons, là où ces derniers ont décidé de passer. Dans l'autre culture, les piétons devront, pour faire la même chose, enjamber quelque pancarte où on aura écrit: «Défense de passer», «Il est interdit de marcher sur l'herbe», etc.

Dans un cas, en culture anglaise, la réalité prime le droit, elle amène à adapter les règles en place; dans l'autre, en culture française ou romano-germanique, le droit précède volontiers la réalité et prétend même la contraindre, parfois au-delà de ce qui est immédiatement nécessaire pour l'ordre de la société.

Cela dit, les textes juridiques passent pour être complexes; qui n'a pas entendu plaisanter ou pester à ce sujet: «Un juriste vous répond et vous ne comprenez déjà plus votre propre question.»

Cela ne tient pas qu'à la complexité des concepts et raisonnements; la méthodologie de même que les habitudes du milieu contribuent à cette complexité. Pour autant, ce n'est pas une exclusivité du domaine juridique.

"J'écris pour compliquer la vie.» nous dit Jerzy Andrzejewsky. «J'écris pour savoir comment j'écris.» semble lui répondre Alberto Moravia.

\section{2) Des cultures et du droit}

Le paramètre culturel — nous revoilà en 1979 - est trop souvent méconnu lorsqu'il est question de droit ou de traduction.

La culture est en effet fondatrice de bien d'autres choses habituellement situées dans d'autres champs de la connaissance. On ne saurait parler utilement du bijuridisme canadien sans évoquer l'enracinement dans leurs cultures d'origine des deux traditions juridiques que nous connaissons. Le droit ayant pour objet de régir l'organisation et les comportements dans la cité, les réflexes culturels qui déterminent le comportement spontané des personnes ne sauraient lui être étrangers.

En fait, le droit est un instrument, une présentation ou une codification des valeurs de la société, une illustration des valeurs que nous avons tirées des religions par exemple. Encore une fois, pour situer la traduction juridique dans son véritable cadre, constatons que le droit reste intimement lié aux cultures et ce, pour plusieurs raisons.

En premier lieu, le droit est attaché à un territoire et, à ce titre, il est influencé, voire enraciné dans la culture du lieu. 
En deuxième lieu, le droit repose en principe sur un consensus sur la manière d'organiser et de faire vivre la cité. Ce consensus est politiquement dégagé et exprimé par des institutions, des lois et des décisions d'État. En cela, le droit est donc second, il est déterminé par une culture; il lui est même ancillaire.

En troisième lieu, le droit est d'abord et indissociablement lié aux questions locales ou privées, ce qui correspond ici grosso modo au droit civil qui régit principalement les personnes, les biens et les obligations. Dans ce domaine au moins, le droit n'invente pas grand chose et ne fait que traduire des valeurs, des coutumes et le bon sens tel qu'il est vécu à travers les époques et les moments par des gens, des familles, et par l'économie, ne l'oublions pas.

En quatrième et dernier lieu, le droit est lié à la culture en ce qu'il s'exprime dans des mots et des raisonnements que les gens doivent comprendre pour pouvoir connaître et appliquer les règles.

Si le droit peut être déterminant, il reste donc dans l'ordre des moyens. Cela aussi, nos étudiants et étudiantes devraient en être instruits.

Rares mais brillants sont les cas où le droit a réussi à devancer la culture. Pensons au domaine de l'assurance automobile au Québec où le législateur a installé en 1978 un régime public d'indemnisation sans égard à la responsabilité. Auparavant, les tribunaux étaient constamment appelés à trancher des situations d'accident dans le simple but de déterminer qui était le responsable. Depuis 1978, on paye sans chercher qui est responsable de l'accident ayant entraîné le dommage corporel. En procédant ainsi, la loi n'a fait qu'interpréter une aspiration sociale fondée avant tout sur les valeurs de solidarité et de compassion, caractéristiques tout à fait remarquables de la société et de la culture québécoises et de l'esprit pratique nord-américain puisque ainsi, on évite les frais, les incertitudes et les délais inhérents au recours aux tribunaux.

C'est un exemple frappant du droit qui se place à l'avant-garde, en éclaireur de l'évolution des esprits, pour traduire de façon articulée et originale de grandes vertus culturelles.

À défaut de tenir compte des paramètres sociaux ou culturels, la rédactrice, le rédacteur, et avec eux les traducteurs et traductrices courent le risque de se couper de leur public cible. Et la loi, texte juridique et de communication, restera sans effet ou encore sera mal comprise, ce qui est évidemment périlleux pour l'ordre social.

Assez parlé de culture, revenons à d'autres aspects caractéristiques de la traduction juridique.

\section{3) Deux langues, quatre vocabulaires}

De la même manière que nous avons dénombré deux cultures et deux systèmes juridiques, le Canada compte également deux langues officielles dans lesquelles s'expriment le droit du Parlement fédéral et celui de certains Parlements provinciaux ou territoriaux.

La traduction des lois est une pratique délicate puisque, comme on vient de le voir plus haut, elle s'inscrit dans un contexte où l'on a un vocabulaire juridique civiliste bilingue et un vocabulaire juridique bilingue de common law. Ce qui fait deux vocabulaires juridiques en français et deux vocabulaires juridiques en anglais.

La traduction juridique met donc en scène beaucoup plus que le droit et la langue à proprement parler. En fait, elle comporte nombre d'enjeux sur lesquels on doit attirer l'attention de nos étudiants et étudiantes. 


\section{Enjeux de la traduction juridique}

On peut distinguer grosso modo des enjeux politiques et des enjeux méthodologiques.

\section{A) Les enjeux politiques}

La traduction juridique est un instrument qui contribue à maintenir symboliquement en équilibre une société partagée en deux langues officielles. C'est ainsi qu'on a pu expliquer l'obligation de traduction systématique ou, plus exactement, le bilinguisme systématique des lois fédérales, québécoises et autres.

Plusieurs ont pensé, au Manitoba par exemple, que ce bilinguisme arrivait trop tard pour aider les minorités de langues officielles. Des juristes francophones ont même laissé entendre qu'ils ne seraient pas portés à invoquer le texte français des lois de telle ou telle province et ce, pour des raisons pratiques. Ces raisons sont parfois contredites par certains succès du programme d'administration de la justice dans les langues officielles ou par les progrès dus aux remarquables efforts de l'Université de Moncton sous la conduite notamment de Gérard Snow, Michel Bastarache et d'autres.

La traduction juridique est un enjeu au plan de la symbolique, mais aussi au plan des batailles et des revendications politiques. Nombre de ces batailles menées par des minorités linguistiques portent, hors du Québec, sur les exigences de bilinguisme dans les textes législatifs ou encore devant tribunaux judiciaires.

Il n'y a pas que des batailles ou des enjeux symboliques avec leurs résonances politiques; sur le plan pratique on doit remarquer que la traduction juridique a permis à certains égards de mettre à la disposition d'une société bilingue des textes nécessaires à son organisation et à son fonctionnement. Sans oublier l'effet des pratiques de traduction juridique jusque sur la fluidité des échanges économiques, en rendant disponibles des instruments juridiques compréhensibles par les intéressés, de part et d'autre de la barrière des langues.

\section{B) Les enjeux méthodologiques}

Tout aussi importants que les enjeux politiques, les enjeux de méthodologie qui s'attachent à la traduction juridique méritent grande attention, dès la conception d'un cours de traduction juridique.

Dans ce domaine, les défis sont multiples. Il ne s'agit pas seulement de transplanter une terminologie précise au fil de phrases ordinaires ou classiques. En traduction juridique on a affaire à des opérations complexes et techniques.

\section{En amont du transfert}

Il s'agit de comprendre le texte de départ, non seulement dans ses mots, mais aussi et surtout dans sa portée. Il faut reconnaître toute sa portée, mais rien que sa portée. Une loi bilingue ou un contrat ne sont pas deux lois ou deux contrats; il s'agit, encore une fois, d'un seul texte en deux langues.

Par ailleurs, le texte juridique est conçu avant tout pour contraindre, permettre ou provoquer des comportements. La loi, par exemple, a principalement pour objet de créer des droits ou des obligations. Ces obligations sont en quelque sorte des 
limites ou des contraintes aux valeurs ou aux libertés des gens. En tradition britannique par exemple, la portée de ces obligations sera donc scrutée non seulement par le législateur qui les édicte, mais aussi par les tribunaux qui les appliquent, les interprètent ou en contrôlent la validité.

Or, dans un système ainsi orienté, la volonté de contrôler la portée des lois ou l'action du législateur a souvent abouti à l'apparition de règles particulières d'interprétation des textes. Face à de telles clefs d'interprétation, le traducteur ou la traductrice doivent considérer que le texte de départ a été conçu non seulement en fonction de son objet, mais aussi en fonction de la façon dont il sera interprété par des tribunaux. Dans un tel contexte, l'entendement des textes juridiques ne peut s'appuyer entièrement sur le sens ordinaire des mots ou sur la façon ordinaire de construire les textes. Il s'agit de comprendre ce qu'a voulu dire le rédacteur, la rédactrice en termes d'effets de droit, sans pouvoir s'appuyer totalement sur le sens courant des mots. On comprend dès lors, pourquoi il faut amener les étudiants et les étudiantes à s'imprégner de la culture juridique, car c'est bien de cela qu'il s'agit, pour comprendre le sens exact du texte de départ. S'agit-il, d'un texte qui a été conçu pour s'appliquer dans un système où il recevra une interprétation libérale ou, au contraire, dans un système où les règles sont interprétées restrictivement, n'ayant alors que le rang de remèdes ponctuels s'appliquant à des situations particulières?

Il faut le savoir. Et donc le dire clairement à nos étudiantes et étudiants. La préparation des traductrices et traducteurs passe donc par la connaissance des prémisses philosophiques, sociologiques et historiques, mais aussi méthodologiques du droit et ce, avant même qu'il ne soit question de traduction.

C'est la raison pour laquelle il est essentiel d'amener les futurs traducteurs ou traductrices à connaître et à comprendre ce qui se passe dans l'esprit du juriste qui a conçu le texte à traduire. Certes, la connaissance de la terminologie bilingue est importante, mais il faut aussi développer la sensibilité de nos étudiants et étudiantes à la différence qui existe dans la manière de rédiger, de formuler ou de comprendre un texte selon la tradition juridique dont il est issu. Cela non plus ne s'invente pas.

\section{Le transfert $d u$ sens}

La seconde opération complexe est bien évidemment celle du transfert du sens, qui suppose une autre série de compétences.

Cela va jusqu'à l'intérêt par exemple de connaître, d'une langue à l'autre, d'un système à l'autre, d'une culture à l'autre, la limite du dit et du non-dit. Cette limite varie selon les cultures. André Gide ne disait-il pas que «Ce qui est le plus précieux en nous est ce qui reste informulé »? D'autres on dit plus récemment qu'en matière juridique, le non-dit n'est pas du non-droit.

Cela demande aussi d'être initié et à la compréhension et au maniement des phrases longues, très fréquentes dans les textes juridiques rédigés en anglais. Il faut aussi avoir réfléchi à l'attitude que les uns et les autres peuvent avoir quant à la déduction, aux associations d'idées, etc. Sans abuser des extrapolations, on peut dire que tout cela touche aux réflexes culturels, voire à l'imaginaire des communautés auxquelles s'adressent les textes de départ et d'arrivée.

En fait, l'objectif est d'amener nos étudiants à être capable de produire un texte d'arrivée qui ressemble le plus possible à un texte rédigé spontanément dans la langue d'arrivée. De la même manière, il faut en fin de compte que les utilisateurs de ces 
textes les comprennent naturellement, de manière à en tirer les mêmes conséquences dans l'une et l'autre langue, en termes de droit, d'obligations et de comportement. C'est dans ce dernier aspect que résident l'essentiel des attentes, de même que le résultat idéal et nécessaire à la fois de la traduction juridique.

\section{Aspects sémantiques}

Il existe également une difficulté supplémentaire: comment s'assurer de la concordance exacte entre les termes qu'on voudra mettre en correspondance? Il existe à cet égard au moins quatre types de contraintes.

D'abord, et sur un plan synchronique, le champ sémantique des termes qu'on pourrait mettre en parallèle d'une langue à l'autre n'est pas toujours exactement le même. Mais cela n'est pas particulier à la langue juridique.

Ensuite, et sur un plan diachronique, l'évolution des deux termes ainsi arrimés ne sera peut-être pas la même dans les deux langues. Ce qui aura été une correspondance exacte à un moment donné ne le sera peut-être plus quelque temps après. On se souviendra que la loi est un texte permanent dont les mots restent, alors que dans la langue courante, ils acquièrent pendant ce temps d'autres nuances, d'autres sens. Là encore, ce n'est pas particulier au bilinguisme juridique.

Troisième type de contrainte, et c'est là que tout se complique, la loi, dès sa rédaction, donne parfois à des mots des sens différents de celui qu'ils ont dans la langue courante et ce, par l'intermédiaire de définitions qui peuvent d'ailleurs varier pour un même mot d'une loi à l'autre. Nous pouvons alors avoir affaire à deux ou quatre sens pour un même mot. D'abord le sens que lui donne la langue courante dans les deux langues concernées; ensuite le sens particulier qu'on voudrait lui donner par la définition qu'on en fait dans la loi elle-même bilingue. Ce qui peut donner jusqu'à quatre sens. Sans compter les variations éventuelles du sens courant dans les deux langues concernées...

Un autre facteur de variation sera le sens que les tribunaux donneront aux mots, en plus du sens courant et de ce qu'on serait porté à comprendre des définitions. Les tribunaux pourront apporter des nuances qui feront autorité par la force de la jurisprudence.

Voilà donc beaucoup de paramètres flottants qui commandent d'être un tant soit peu au courant des aspects particuliers du droit lui-même et de ses méthodes tout aussi particulières.

Compte tenu de l'ensemble de ces contraintes, il est utile de prévenir nos étudiants qu'il existe autant de chances d'établir un lien parfait entre deux termes que de passer d'une montgolfière à une autre en marchant sur une corde tendue entre les deux nacelles. On comprend que ce doit être un moment rare, un peu comme le moment où, dans un regard, deux êtres se rejoignent et où, comme le dit Alfred de Musset, «Il n'est pas besoin de lire ce que disent les lèvres quand on écoute les cœurs se répondre.» Combien de temps cette corde tendue entre les deux nacelles conservera-t-elle son inflexible tension?

«Il n'est pas certain que tout soit certain.» nous dirait Blaise Pascal. Un autre, Diogène Laërce, nous dirait que «Le vrai n'est pas plus sûr que le probable». Bertrand Russell achèverait de nous dérouter, lui qui a dit que «Ce que les hommes veulent en fait, ce n'est pas la connaissance, c'est la certitude.» 
En fait, le traducteur doit avoir une approche téléologique: il transfère non pas des mots, mais des effets de droit, un résultat escompté, ce qui suppose qu'il soit en mesure de comprendre les objectifs poursuivis par le rédacteur du texte de départ. C'est sans doute la clef d'un vieux débat entre les tenants de la traduction littérale et les tenants de la traduction téléologique, c'est-à-dire de la traduction visant à produire un effet équivalent dans la langue d'arrivée. Trop longtemps, la traduction littérale, au nom d'une certaine conception de la fidélité, a en fait condamné le lecteur du texte d'arrivée à devoir connaître le texte de départ pour pouvoir comprendre la traduction. C'est la négation même de l'utilité du traducteur et de la traductrice. Parlons donc, de l'utilité du traducteur.

\section{De l'utilité des traducteurs et de la traduction}

Le traducteur et la traductrice ont longtemps été perçus comme des personnes arrivant tout à la fin du processus, alors qu'il s'agit de réaliser en urgence et parfois pour la forme, la traduction d'un texte déjà rédigé. Un moment où les choses sont trop souvent présentées comme déjà "attachées », «bouclées ». Cette façon de faire est une erreur de méthode.

Le traducteur, la traductrice ne sont pas des machines. Ils ne sont pas non plus des secrétaires bilingues. Ils et elles sont des professionnels et sont, en plus, des collègues très précieux pour la vérification de la qualité du texte juridique et ce, avant même qu'ils se mettent à traduire.

En effet, le traducteur est avant tout un lecteur très attentif du texte de départ. Il est même souvent le premier vrai lecteur du texte de départ. Pensons-y; le texte de départ résulte la plupart du temps de discussions entre techniciens ou politiciens qui ont déjà en quelque sorte «négocié » son contenu. Ils le connaissent par cœur et leur regard n'a peut-être plus l'acuité qu'il faudrait pour vérifier eux-mêmes si la façon dont il est rédigé livrera bien le sens voulu à un lecteur qui, lui, le découvre.

Le traducteur, la traductrice ont un regard neuf, comme le citoyen ou le juge qui auront à appliquer le texte. Ils sont obligés d'en comprendre toutes les nuances, puisqu'ils devront les répercuter dans l'autre langue. On l'a compris, le traducteur ou la traductrice seront donc de bons réviseurs et de bons observateurs du texte. Et ce, avant même de réaliser la traduction elle-même.

Ainsi, la traduction met en scène des compétences qui interviennent et sont utiles bien en amont du transfert de sens lui-même. Ce qui précède la traduction (étude $\mathrm{du}$ sens et de la logique du texte, recherche terminologique) est une intervention professionnelle essentielle. Le texte de départ a-t-il fait l'objet d'autant d'attention et de vérification dans sa préparation même?

Comme l'a dit un poète libanais, «L'élan fait partie du saut».

S’interrogeant sur la raison pour laquelle ses films ont plus de succès en Europe qu'aux États-Unis, son propre pays, le cinéaste Woody Allen formulait d'ailleurs l'hypothèse toute personnelle selon laquelle ses films avaient certainement gagné quelque chose lors de la traduction («Wild man blues», 1998)...

\section{Les moyens}

Dans cette dernière série de considérations, réfléchissons rapidement à deux sujets principaux : faut-il être juriste pour aborder la traduction juridique? Faut-il faire une 
maîtrise orientée en traduction juridique pour toucher à ce domaine? Enfin, la formation en traduction juridique telle qu'on la connaît actuellement est-elle adéquate?

Tout d'abord, faut-il être juriste pour traduire des textes juridiques ou peut-on aborder ce domaine avec une formation de traducteur? C’est un vieux débat qui subsiste non seulement pour le droit, mais également pour d'autres disciplines où il est courant d'entendre dire que seul un ingénieur pourrait traduire des textes reliés au génie, seul un médecin pourrait traduire des textes à teneur médicale etc.

Même si ces suppositions paraissent d'une certaine logique, il ne faut pas nier la compétence professionnelle des traducteurs et traductrices que nous formons dans nos programmes. Le cadre d'un programme de trente cours au niveau universitaire permet de donner à ces personnes une formation solide qui, dans l'idéal, les prépare ou pourrait les préparer à aborder les domaines les plus divers sans avoir à faire à chaque fois les efforts d'une formation complète dans la discipline du texte.

Allons plus loin. Lorsqu'il s'agit de traduire, la clef de la qualité est avant tout l'aptitude à la traduction. En effet, une bonne traduction ne s'improvise pas et il n'est pas évident qu'il suffirait d'être juriste ou psychologue bilingues pour pouvoir faire de la traduction de niveau professionnel dans ces domaines.

La traduction, en droit comme ailleurs, doit être assise sur des compétences particulières en matière linguistique comme en matière de communication. Un texte, juridique ou non, est avant tout un instrument de communication. Il n'est pas si clair que des juristes ou des médecins par exemple aient toujours les aptitudes à la communication qui garantiraient aux lecteurs et aux lectrices un texte d'arrivée compréhensible.

Pour reprendre un instant ce que nous évoquions plus haut quant à l'apport particulier des traducteurs dans la révision du texte de départ, on peut noter que nombre d'erreurs importantes voire essentielles ont pu être décelées dans les textes juridiques au stade de la traduction lorsqu'on a fait appel à des traducteurs et traductrices bien formés, dont on a la sagesse d'écouter les observations et les conseils.

Bref, la compétence en matière de traduction est avant tout celle du traducteur et de la traductrice. Cela non plus ne s'improvise pas. Il convient de se rappeler qu'au Québec par exemple les traducteurs, terminologues et interprètes sont une profession encadrée en vertu de la loi et qu'un ordre professionnel la réglemente pour garantir au public la compétence et l'intégrité des membres. Cela étant, ces professionnels doivent, dans la plénitude de leur pratique, tenir compte de certaines limites que constituent les activités réservées par la loi à d'autres professionnels dans le domaine du droit, de la santé, du génie et de l'aménagement ou encore des affaires.

\section{La formation}

La formation des traducteurs et traductrices juridiques est-elle adéquate au premier cycle? On nous pardonnera de poser la question de façon aussi brutale. Les professionnels de la traduction au Québec constituent un pôle d'excellence, certes. Mais vingt-cinq ans d'enseignement amènent à penser que nos traducteurs et traductrices pourraient être mieux formés en traduction juridique. Quarante-cinq heures ne suffisent sans doute pas. Il y a peut-être même une question de responsabilité pour les universités dans la mesure où, en mettant au programme un cours de traduction dans 
une spécialité donnée, nos établissements laissent entendre aux étudiants et étudiantes qu'ils pourraient, au terme des quarante-cinq heures, avoir acquis une compétence suffisante pour faire de la traduction dans cette spécialité.

Comme on l'a vu, le domaine juridique est multiforme. Mais on se rend compte également du fait que pour avoir une chance de comprendre la portée du texte à traduire, il faut avoir réfléchi à nombre de phénomènes dans lesquels le droit plonge ses racines. Un texte juridique se situe déjà à un niveau élevé d'abstraction. Les concepts en cause ne sont pas toujours définis comme une figure géométrique tracée sur une feuille de papier. Le droit charrie nombre de valeurs et de notions molles qu'il faudra comprendre en contexte, selon l'esprit du rédacteur ou de la rédactrice.

En quarante-cinq heures et malgré les préalables imposés pour l'accès à ce genre de cours, il est difficile de couvrir les aspects théoriques et pratiques qu'il serait pourtant utile d'aborder. C'est pourquoi, depuis plus de vingt ans, nous avons fait certains choix stratégiques et décidé de traiter en priorité trois aspects.

En premier lieu, l'objectif du cours est de présenter le phénomène juridique pour ce qu'il est, c'est-à-dire selon sa finalité dans la société, mais également selon son origine et son ancrage dans la philosophie, dans la sociologie et dans l'univers politique.

En deuxième lieu, comme on l'a vu, nous avons choisi de décrire sommairement les traits caractéristiques de la dualité juridique canadienne, avec comme ambition d'amener les étudiants et les étudiantes à comprendre ce qui se passe dans l'esprit d'un juriste de tradition britannique ou de tradition civiliste lorsqu'ils écrivent, ou lorsqu'ils interprètent une loi. Le but est simple: il s'agit de s'assurer que, pendant son activité de traduction, le traducteur ou la traductrice auront les clefs qu'il faut pour reconnaître par exemple la conception de la loi dont il s'agit: selon le système, aura-t-on affaire à une loi simplement correctives ou à une loi que nous appellerions "programmatoire»?

En troisième lieu, nous mettons l'accent sur des phénomènes de rédaction. En effet, il semble clair que les principales difficultés d'ordre technique qui se présentent au traducteur ou à la traductrice sont liées à des phénomènes de rédaction, au-delà des phénomènes de terminologie. Ne disposant que de quarante-cinq heures, on doit considérer que les étudiants et étudiantes auront accès à des instruments terminologiques, alors que pour la rédaction, il leur aura fallu développer des attitudes et des aptitudes qui ne se trouvent pas au tournant d'une page, dans un dictionnaire ou dans une grammaire. Il s'agit par exemple des difficultés souvent rencontrées dans des textes anglais, telles les structures alphanumériques complexes, les phrases longues, les protocoles de définition, etc.

Certes il pourrait être intéressant d'amener les étudiants et les étudiantes dans une sorte de visite guidée des différents domaines du droit, en leur faisant aborder des textes différents, en leur faisant faire des recherches terminologiques dans ces domaines. Compte tenu du cadre classique des quarante-cinq heures, nous avons estimé depuis 1974 que ce cadre est trop restreint et qu'il est prioritaire de s'assurer qu'ils auront une compréhension générale du phénomène juridique, de la culture spécifique des juristes qui rédigent, de même que les cléfs suffisantes pour le maniement de ces textes complexes. 


\section{Conclusion}

On peut faire de la traduction juridique.

La traduction juridique est une activité de niveau professionnel qui devrait être confiée à des personnes, juristes ou non-juristes, qui ont une bonne formation en traduction.

Le programme de traduction devrait mieux refléter les caractéristiques et contraintes particulières de la traduction juridique. Concrètement, cela signifie que le cadre consacré à cette matière dans le programme de premier cycle aurait avantage à être agrandi. Si nous osions nous commettre avec un sens raisonnable de l'économie, on pourrait dire qu'un bloc de 90 heures réparti en deux cours permettrait déjà de mieux répondre aux exigences du domaine.

Un premier cours serait principalement consacré à la présentation des phénomènes juridiques. Pensons d'abord aux aspects socio-historiques, politiques et culturels du droit, pour aborder ensuite les questions de méthodologie juridique, en terminant par une initiation soigneusement planifiée aux principales difficultés que proposent d'ordinaire la rédaction et l'interprétation des textes juridiques.

Une fois cette base assurée, le second cours pourrait être consacré, comme le sont la plupart des cours de traduction juridique, à une initiation à la traduction et à la terminologie juridiques par secteurs. Ce second cours permettrait de proposer aux étudiants et aux étudiantes des textes plus spécialisés, plus appliqués.

Comme on le voit, ces considérations et suggestions ne disposent pas de l'ensemble des aspects académiques ou pédagogiques de la traduction juridique. Bien des choses resteraient à dire. Rendez-vous (en 2025 ?) pour le troisième numéro thématique de Meta sur la traduction juridique...

\section{Du même auteur sur ce sujet}

Pour une dimension culturelle de la traduction juridique, Meta, vol. 24, nº 1, PUM, mars 1979, $27 \mathrm{p}$.

Loi et héritage culturel, Les Cahiers de droit, mars 1979, 30 pages, Presses de l’Université Laval, Québec.

Propos sur la rédaction des lois, Conseil de la langue française, 245 pages, août 1979, Éditeur officiel du Québec.

Guide de la traduction appliquée, coauteurs Geoffrey Vitale et Robert Larose, Tome I, 496 pages, 1979, Presses de l'Université du Québec.

Deux langues, deux cultures: deux lois, Le Devoir, quotidien, Montréal, 11 février 1980, page 5.

Rédaction des lois, rendez-vous du droit et de la culture, coauteur Wallace J. Schwab, 350 pages, Conseil de la langue française, juin 1980 Québec, Éditeur officiel du Québec (troisième tirage, 1997).

Guide de la traduction appliquée, Geoffrey Vitale et Robert Larose, Tome II, 500 pages, Presses de l'Université du Québec, novembre 1980.

La rédaction française des lois, coauteurs Marie Lajoie, Wallace J. Schwab, Jean Côté, 200 pages, Commission de réforme du droit du Canada, 1980.

Stéréophonie législative: des risques de haute infidélité, Les Cahiers de droit, Québec, mars 1981, 32 pages, Presses de l'Université Laval.

Rédaction des lois, langage et valeurs: les enjeux in Langage du droit et traduction, collectif, 320 pages, p. 111-121, Conseil de la langue française, Québec, Éditeur officiel du Québec, 1982. 
La boulimie des lois, Le Soleil, quotidien, Québec, janvier 1983.

Elizabeth, que de lois en Ton nom!, revue Circuit, n 3, mars 1984, Montréal.

Le citoyen du Québec dans tous ses États (I, II, III) 15, 16 et 17 mars 1984, Le Soleil, quotidien, page éditoriale (Québec).

Traduction des textes législatifs, contraintes et enjeux, in Traduction et qualité de langue, coll., 220 pages, p. 98-104, Conseil de la langue française, Québec, 1984.

Droits collectifs: la méprise, Le Devoir, quotidien, Montréal, 29 mai 1985, p. 9. 